\title{
HIPERENDEMIA DE EQUINOCOCOSIS Y FERTILIDAD QUÍSTICA EN PORCINOS DEL VALLE INTERANDINO DE HUANCARAMA, PERÚ
}

\author{
Rony Sierra-Ramos ${ }^{1, a}$, Aldo Valderrama-Pomé $2, b$
}

\begin{abstract}
RESUMEN
El objetivo de la investigación fue determinar el porcentaje de infección de equinococosis en porcinos, fertilidad quística y factores asociados. Estudio de corte transversal. La población estuvo conformada por 579 porcinos faenados en el matadero de Huancarama. Los quistes hidatídicos fueron detectados mediante evaluación post mórtem. La fertilidad se determinó midiendo el diámetro de los quistes y observando microscópicamente la membrana germinativa en busca de protoescólices. El porcentaje de infección de equinococosis quística fue de 76,7\% (IC95\%: 73,2-80,3). Los porcinos mayores a dos años tuvieron más oportunidad de presentar quistes (RP 1,03; IC95\%:1,0-1,1; $p<0,01$ ), al igual que los provenientes del distrito de Andahuaylas (RP 1,2; IC95\%: 1,1-1,4; p<0,01). Los quistes pulmonares presentaron menor porcentaje de infección que los hepáticos. Se examinaron 346 quistes hidatídicos de 334 porcinos, se encontró una fertilidad total de 42,8\% (IC95\%: 37,4-48,1).
\end{abstract}

Palabras clave: Equinococosis, fertilidad, porcinos (fuente: DeCS BIREME).

\section{ECHINOCOCCOSIS HYPER-ENDEMIC AND CYSTIC FERTILITY IN PIGS OF THE INTER-ANDEAN VALLEY OF HUANCARAMA, PERU}

\begin{abstract}
The objective of the research was to determine the percentage of echinococcosis infection in swine, cystic fertility and associated factors. Cross-sectional study. The population was formed by 579 pigs slaughtered in the slaughterhouse of Huancarama. Hydatid cysts were detected by postmortem evaluation. Fertility was determined by measuring the diameter of the cysts and microscopically observing the germinative membrane in search of protoscoleces. The percentage of cystic echinococcosis infection was 76.7\% (Cl 95\%: 73.2-80.3). Pigs older than two years were more likely to have cysts (RP 1.03; $\mathrm{Cl} 95 \%$ : 1.0-1.1; ( $<$ < 0.01), as well as those coming from the Andahuaylas district (RP 1.2; Cl 95\%: 1.1-1.4; $\mathrm{P}<0.01)$. Lung cysts showed a lower percentage of infection than hepatic ones. We examined 346 hydatid cysts of 334 pigs, a total fertility of $42.8 \%$ was found (Cl $95 \%$ : $37.4-48.1)$.
\end{abstract}

Key words: Echinococcosis, fertility, swine (source: MeSH NLM).

\section{INTRODUCCIÓN}

La equinococosis es una enfermedad zoonótica crónica causada por el estado larvario del Echinococcus, endoparásito perteneciente a la clase Cestoda, familia Taeniidae, que infecta animales domésticos, salvajes y también a seres humanos ${ }^{(1,2)}$. De las cinco especies, $E$. granulosus y $E$. multilocularis son los de mayor riesgo zoonótico ${ }^{(3)}$. El parásito se transmite entre perros y varias especies unguladas domésticas. El estado larvario consiste en un quiste hidatídico unilocular. El crecimiento es expansivo y puede alcanzar un diámetro de hasta $30 \mathrm{~cm}$, especialmente en hígado y pulmones, lo cual se denomina equinococosis quística (1).
En América, Perú es el país con mayor infección (2). Huancarama es zona endémica donde la especie más afectada es la porcina (4). Los animales infectados disminuyen la producción de carne y sus vísceras son decomisadas, ocasionando pérdidas económicas millonarias. El hombre cierra el ciclo biológico del parásito alimentando al perro con vísceras crudas con quistes ${ }^{(5)}$.

Los quistes se clasifican en fértiles e infértiles ${ }^{(6)}$. Los fértiles producen protoescólices y son capaces de continuar el ciclo biológico del parásito infectando al hospedero definitivo ${ }^{(3)}$. Por lo tanto, el objetivo del estudio fue determinar el porcentaje de infección de equinococosis en porcinos, fertilidad quística y factores asociados en Huancarama, Perú.

\footnotetext{
Universidad Nacional Micaela Bastidas, Abancay, Perú.

Laboratorio de Patología Clínica. Universidad Nacional Micaela Bastidas, Abancay, Perú.

Médico veterinario y zootecnista; ${ }^{b}$ médico veterinario y zootecnista, magister scientiae en Salud Pública.
}

Los datos de este artículo forman parte de la tesis de Rony Sierra-Ramos para obtener el grado de Médico Veterinario y Zootecnista en la Universidad Nacional Micaela Bastidas.

Recibido: 14/11/2016 Aprobado: 19/04/2017 En línea: 28/06/2017

Citar como: Sierra-Ramos R, Valderrama-Pomé A. Hiperendemia de equinococosis y fertilidad quística en porcinos del valle interandino de Huancarama, Perú. Rev Peru Med Exp Salud Publica. 2017;34(2):250-4. doi: 10.17843/rpmesp.2017.342.2500 


\section{MATERIALES Y MÉTODOS}

Huancarama es uno de los 19 distritos de la provincia de Andahuaylas, región Apurímac (Figura 1). Ubicado a 2980 metros de altitud entre las coordenadas $13^{\circ} 38^{\prime} 48^{\prime}$ latitud Sur y $73^{\circ} 05^{\prime} 08^{\prime}$ longitud Oeste. Las principales actividades económicas son la agricultura y la ganadería. Es una de las zonas más pobres de Perú, con $79,5 \%$ de pobreza ${ }^{(7)}$. El estudio se realizó en el matadero de Huancarama durante junio y julio de 2013, temporada de mayor faenamiento y consumo de cerdo, debido a las celebraciones de aniversario distrital y Fiestas Patrias. El matadero solo cumple con $20 \%$ de las condiciones sanitarias inherentes a instalaciones y prácticas del personal, dispuestas por el Servicio Nacional de Sanidad Agraria (SENASA) de Perú (8).

Se realizó un estudio transversal analítico. La población estuvo conformada por la totalidad de porcinos (579) faenados en el matadero durante los meses señalados. Se preguntó a los acopiadores acerca del lugar de procedencia de los porcinos que benefician, información que se obvia en el cuaderno de registro del matadero. Luego del faenado se determinó la edad de los animales mediante cronología dentaria, clasificándolos en $<2$ años (jóvenes) y $\geq 2$ años (adultos). Los quistes fueron detectados mediante evaluación post mórtem (fase obligatoria del faenado) que comprende el examen visual, palpación e incisión de vísceras, de acuerdo al Reglamento Sanitario del Faenado de Animales de Abasto (9).

Para determinar la fertilidad se escogieron quistes hepáticos y pulmonares mayores a $10 \mathrm{~mm}$ de diámetro ${ }^{(10)}$. En el Centro de Salud de Huancarama se midieron los quistes

\section{MENSAJES CLAVE}

Motivación para realizar el estudio. El distrito de Huancarama es endémico de equinococosis quística, donde la especie más afectada es la porcina.

Principales hallazgos. El porcentaje de infección por equinococosis quística fue de $76,7 \%$. Los porcinos mayores a dos años tuvieron más oportunidad de presentar quistes, al igual que los provenientes del distrito de Andahuaylas. Los quistes pulmonares presentaron menor porcentaje de infección que los hepáticos. Se encontró una fertilidad de $42,8 \%$.

Implicancias. Se requiere realizar intervenciones a fin de reducir la frecuencia de equinococosis en poblaciones endémicas.

con una regla milimetrada, clasificándolos en $<2 \mathrm{~cm}$ y 2-10 cm. Se les realizó una punción con jeringa estéril para eliminar la presión intraquística, luego se abrió el quiste para extraer la membrana germinativa. Se observó microscópicamente, a un aumento de 40X, la presencia o ausencia de protoescólices para clasificar a los quistes como fértiles o infértiles. El análisis estadístico se efectuó con el paquete SPSS 23. Se realizó el análisis univariado para determinar frecuencias y porcentajes, y el bivariado para determinar posibles asociaciones, se usó razones de prevalencia e intervalos de confianza al $95 \%$ con un a de 0,05 .

\section{RESULTADOS}

De 579 porcinos faenados, $444(76,7 \%)$ presentaron equinococosis (IC95\%: 73,2-80,2). La Tabla 1 muestra
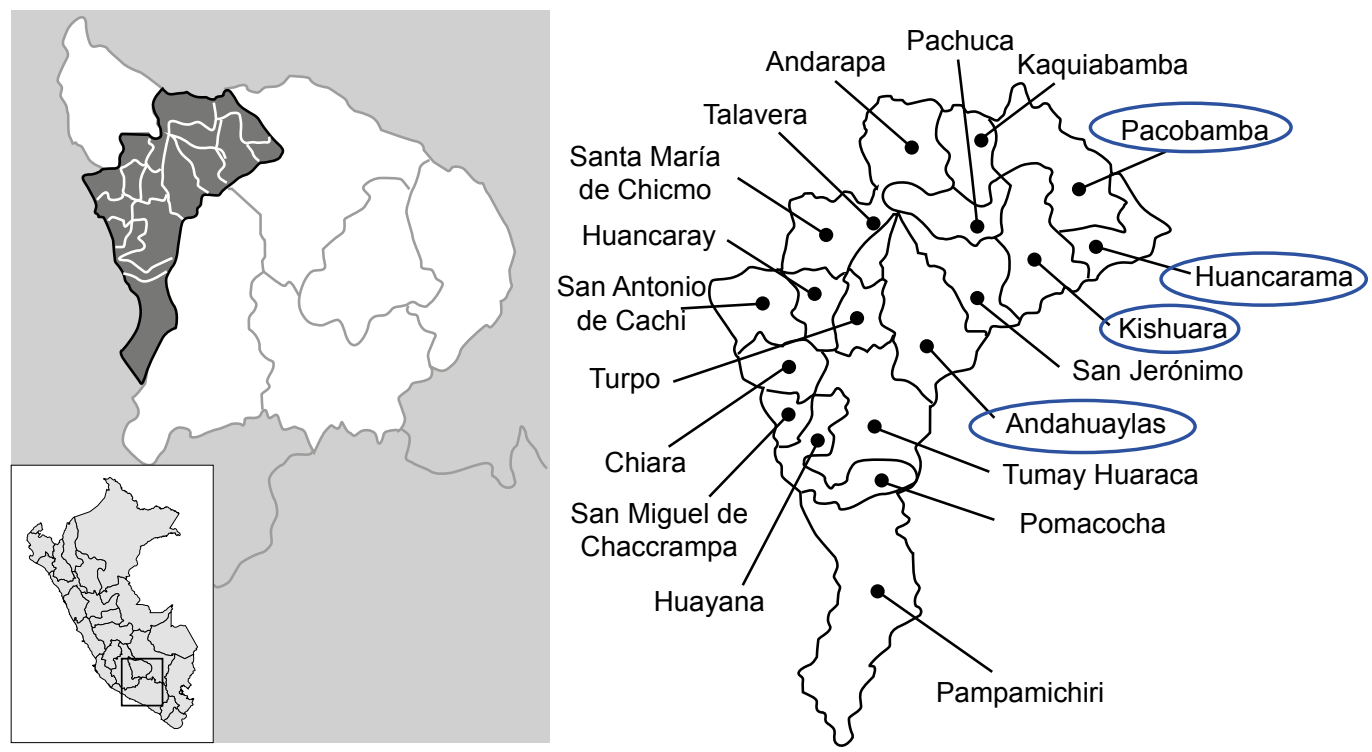

Figura 1. Procedencia de los porcinos faenados en el matadero del distrito de Huancarama, provincia de Andahuaylas, región Apurímac, durante junio y julio de 2013 
Tabla 1. Porcentaje de infección de equinococosis quística en porcinos faenados en el matadero de Huancarama durante junio y julio de 2013 y factores asociados

\begin{tabular}{|c|c|c|c|c|}
\hline Factores asociados & $\begin{array}{l}\text { Porcinos con equinococosis } \\
(444 / 579)\end{array}$ & $\mathbf{R P}$ & IC (95\%) & Valor $p$ \\
\hline \multicolumn{5}{|l|}{ Sexo del porcino } \\
\hline Hembra & $174 / 222(78,4)$ & 1 & & \\
\hline Macho & $270 / 357(75,6)$ & 0,965 & $0,881-1,057$ & 0,441 \\
\hline \multicolumn{5}{|l|}{ Edad del porcino } \\
\hline$<2$ años & $234 / 323(72,4)$ & 1 & & \\
\hline$\geq 2$ años & $210 / 256(82,0)$ & 1,032 & $1,009-1,055$ & 0,006 \\
\hline \multicolumn{5}{|c|}{ Procedencia del porcino } \\
\hline Huancarama & $337 / 448(75,2)$ & 1 & & \\
\hline Pacobamba & $74 / 89(83,1)$ & 1,105 & $0,992-1,238$ & 0,068 \\
\hline Kishuara & $20 / 28(71,4)$ & 0,949 & $0,747-1,208$ & 0,673 \\
\hline Andahuaylas & $13 / 14(92,9)$ & 1,234 & $1,057-1,441$ & 0,008 \\
\hline \multicolumn{5}{|l|}{ Localización del quiste } \\
\hline Hepática & $407 / 579(70,3)$ & 1 & & \\
\hline Pulmonar & $135 / 579(23,3)$ & $9,63 e-09$ & $8,13 e-09-1,14 e-08$ & $p<0,001$ \\
\hline Hepatopulmonar & $37 / 579(6,4)$ & 1 & $0,999-1,000$ & 1,000 \\
\hline
\end{tabular}

RP: Razón de prevalencia, IC: Intervalo de confianza

que los porcinos $>2$ años tuvieron más probabilidad de presentar quistes, al igual que los provenientes de Andahuaylas. Además, los quistes pulmonares presentaron menor porcentaje de infección. El sexo no mostró asociación con la infección. La equinococosis en $<2$ años y $\geq 2$ años de ambos sexos tampoco resulto asociada. El porcentaje de fertilidad de quistes no mostró asociación con la edad y el sexo $\left(X^{2}=0,064 ; p=0,801\right)$, lo que demuestra que no hay sesgo en los resultados y se mantienen los porcentajes de infección.
Se examinaron 346 quistes de 334 porcinos, encontrándose una fertilidad de 42,8\% (IC95\%: 37,4$48,1)$. Los quistes hepáticos tuvieron una fertilidad de $42,9 \%$ (IC95\%: 37,4-48,6) y los pulmonares 40,6\% (IC95\%: 22,1-59,2). La Tabla 2 muestra que las hembras presentan mayor frecuencia de quistes hepáticos fértiles, al igual que los porcinos $\geq 2$ años. El menor porcentaje de quistes hepáticos fértiles provino del distrito de Pacobamba. Los quistes hepáticos y pulmonares de 2-10 cm presentaron mayor porcentaje

Tabla 2. Fertilidad de quistes hidatídicos en vísceras de porcinos faenados en el matadero de Huancarama durante junio y julio de 2013 y factores asociados

\begin{tabular}{|c|c|c|c|c|c|c|c|c|}
\hline $\begin{array}{l}\text { Factores } \\
\text { asociados }\end{array}$ & $\begin{array}{c}\text { Quistes } \\
\text { hepáticos } \\
\text { fértiles } \\
(135 / 314)\end{array}$ & RP & IC(95\%) & Valor $p$ & $\begin{array}{c}\text { Quistes } \\
\text { pulmonares } \\
\text { fértiles } \\
(13 / 32)\end{array}$ & RP & IC(95\%) & Valor $p$ \\
\hline \multicolumn{9}{|l|}{ Sexo } \\
\hline Hembra & $86 / 140(61,4)$ & 1 & & & $5 / 12(41,7)$ & 1 & & \\
\hline Macho & $49 / 174(28,2)$ & 0,458 & $0,349-0,601$ & $p<0,001$ & $8 / 20(40,0)$ & 0,96 & $0,407-2,264$ & 0,926 \\
\hline \multicolumn{9}{|l|}{ Edad } \\
\hline$<2$ años & $23 / 112(20,5)$ & 1 & & & $2 / 10(20,0)$ & 1 & & \\
\hline$\geq 2$ años & $112 / 202(55,4)$ & 2,699 & $1,838-3,967$ & $p<0,001$ & $11 / 22(50,0)$ & 2,5 & $0,676-9,248$ & 0,170 \\
\hline \multicolumn{9}{|l|}{ Procedencia } \\
\hline Huancarama & $48 / 93(51,6)$ & 1 & & & $2 / 10(20,0)$ & 1 & & \\
\hline Pacobamba & $64 / 174(36,8)$ & 0,713 & $0,540-0,939$ & 0,016 & $5 / 14(35,7)$ & 1,786 & $0,429-7,424$ & 0,425 \\
\hline Kishuara & $18 / 28(64,3)$ & 1,246 & $0,887-1,748$ & 0,204 & $5 / 7(71,4)$ & 3,571 & $0,949-13,439$ & 0,060 \\
\hline Andahuaylas & $5 / 19(26,3)$ & 0,509 & $0,234-1,097$ & 0,090 & $1 / 1(100,0)$ & 1 & - & - \\
\hline \multicolumn{9}{|l|}{ Diámetro } \\
\hline$<2 \mathrm{~cm}$ & $22 / 123(17,9)$ & 1 & & & $4 / 22(18,2)$ & 1 & & \\
\hline $2-10 \mathrm{~cm}$ & $113 / 191(59,2)$ & 3,308 & $2,223-4,921$ & $p<0,001$ & $9 / 10(90,0)$ & 4,95 & $1,992-12,299$ & 0,001 \\
\hline
\end{tabular}

RP: Razón de prevalencia, IC: Intervalo de confianza 
de fertilidad. El sexo, la edad y la procedencia de los quistes pulmonares no mostraron asociación con la fertilidad. No se encontraron quistes $>10 \mathrm{~cm}$. El porcentaje de fertilidad de quistes entre las variables edad y sexo no mostró asociación $\left(X^{2}=3,791 ; p=0,052\right)$, lo que demuestra que no hay sesgo en los resultados $y$ se mantienen los porcentajes de fertilidad.

\section{DISCUSIÓN}

El porcentaje de infección con equinococosis $(77 \%)$ en esta investigación es muy superior al de otras especies y viene incrementándose los últimos años, debido a que los criadores de porcinos faenados en Huancarama muestran deficientes conocimientos y prácticas preventivas de zoonosis; tal es así que, el 2011 se reportó un porcentaje de infección de $72 \%{ }^{(4)}$.

Este porcentaje es variable, en Junín se halló de 14$88 \%$ (11); en Cusco de $37-64 \%$ y en Calca un $44 \%$. Igualmente, en otros países, como México, se reportó de 0,3-7\% ${ }^{(12,10)}$; Argentina de $2-12 \%{ }^{(13)}$ y Chile de $4-48 \%{ }^{(14)}$. Las diferencias de porcentajes de infección entre ciudades podrían deberse a condiciones higiénicas de los mataderos, condiciones climatológicas, tasa de contaminación de los perros, forma de faenamiento y estado nutricional de los animales.

Los porcinos $<2$ años presentaron menor porcentaje de infección (72\%), lo que se debería al poco tiempo de exposición a los factores de riesgo ${ }^{(6)}$; esto explicaría las altas tasas de equinococosis en mataderos rurales, ya que en estos faenan animales de edad avanzada.

Los porcinos con mayor porcentaje de infección provinieron del distrito de Andahuaylas (93\%), coincidiendo con reportes previos del lugar (4). Esto podría deberse a que en las zonas de procedencia de los porcinos predomina la "crianza de traspatio" y se desconoce el papel que desempeñan en la zoonosis ${ }^{(12)}$.

El alto porcentaje de quistes hepáticos hallado (70\%), podría deberse a que el embrión hexacanto atraviesa la mucosa intestinal y pasa a la circulación portal hacia el hígado, que se comporta como el primer filtro. La localización y desarrollo de los quistes parecen estar relacionadas con características anatómicas y fisiológicas del hospedador, así como la especie y cepa del parásito, por lo que, en porcinos se ubican preferentemente en hígado y pulmones ${ }^{(15)}$. Este resultado coincide con un estudio de la zona, donde se reportó $41 \%$ de quistes hepáticos, $25 \%$ de hepatopulmonares y $5 \%$ de pulmonares ${ }^{(4)}$. También, coincide con reportes de Argentina con $98 \%$ de quistes hepáticos, $5 \%$ de hepatopulmonares y $2 \%$ de pulmonares ${ }^{(13)}$. En México, los quistes hepáticos prevalecieron entre $93-99 \%{ }^{(10,12)}$. En Chile se reportó $80 \%$ de quistes hepáticos y $11 \%$ de pulmonares ${ }^{(14)}$.

No se encontró asociación entre el sexo y la presencia de quistes, igual a lo reportado en Argentina ${ }^{(13)}$. El porcentaje de infección de equinococosis en $<2$ años y $\geq 2$ años no estuvo asociado al sexo.

El porcentaje de fertilidad encontrado fue alto (43\%). La fertilidad de quistes puede deberse a factores ecológicos, variaciones en la composición aminoacídica del líquido hidatídico o a la presencia de subespecies de Echinococus granulosus ${ }^{(6)}$, ya que existen evidencias de que en muchas áreas endémicas del mundo circulan variantes intraespecíficas o cepas con patrones de desarrollo diferentes que, en conjunto, afectan la epidemiología del parásito ${ }^{(15)}$. Similares resultados se hallaron en India con $40 \%{ }^{(16)}$, solo superados por Kenia con $80 \%{ }^{(17)}$. Sin embargo, existen reportes inferiores como en México con $35 \%{ }^{(12)}$, Argentina con $22 \%{ }^{(13)}$ y Eslovaquia con $25 \%{ }^{(18)}$.

El elevado porcentaje de quistes hepáticos fértiles en hembras $(61 \%)$ podría deberse a que en ellas se encontraron quistes de mayor tamaño. Además, la respuesta de anticuerpos a la infección por Equinococus sp. es anterior a la producción de líquido hidatídico, lo que evidencia que la activación, movilidad e instalación en los tejidos de la oncósfera, generan respuestas del sistema inmunitario del hospedero (19). Las inmunoglobulinas producidas por el hospedero penetran las células de la capa germinal, se ubican mayoritariamente en el núcleo, impiden la formación de yemas y protoescólices e inhiben la proliferación celular o induce apoptosis. Así mismo, la inmunidad natural podría impedir el desarrollo y crecimiento de protoescólices y mantener el quiste en un estado infértil, lo que en las hembras podría verse mermado, debido a mecanismos inmunodepresores durante la parición ${ }^{(20)}$. El porcentaje de fertilidad de quistes en $<2$ años $y \geq 2$ años no estuvo asociado al sexo.

El alto porcentaje de quistes hepáticos fértiles en porcinos $\geq 2$ años (55\%) se debería a que los quistes desarrollan a razón de $1 \mathrm{~cm}$ de diámetro por año, por lo que se hacen más evidentes en animales de edad avanzada ${ }^{(10)}$.

El porcentaje de quistes fértiles hepáticos y pulmonares de $2-10 \mathrm{~cm}$, encontrado en este estudio fue elevado (59 y 90\%, respectivamente), con lo que se evidencia que a mayor diámetro se incrementa la fertilidad ${ }^{(6)}$. En nuestra investigación no se encontraron quistes $>10 \mathrm{~cm}$, posiblemente porque su crecimiento está limitado por la corta vida de los porcinos, en comparación a la humana. El crecimiento del quiste dependerá del potencial evolutivo del embrión hexacanto, del tejido circundante y de la resistencia del huésped ${ }^{(19)}$. Así mismo, el tamaño de los quistes depende del órgano donde se alojan ${ }^{(2)}$. Además, los animales $\geq 2$ años están más parasitados, tienen quistes de mayor tamaño y en mayor número, en comparación con los animales <2 años. Nuestros resultados concuerdan con investigaciones de Argentina y México, donde se halló asociación significativa entre el tamaño y la fertilidad de los quistes ${ }^{(12,13)}$. Así mismo, en Eslovaquia, determinaron que 
el tamaño de los quistes fértiles fue tres veces mayor al diámetro de los quistes infértiles ${ }^{(18)}$.

Entre las limitaciones de nuestro estudio podemos mencionar que no fue posible aplicar un cuestionario a los acopiadores o propietarios de los porcinos faenados, para recabar más factores asociados, debido a que se mostraron celosos de evidenciar infecciones en sus animales, lo cual generaría decomiso de vísceras y la consiguiente pérdida económica. Por otro lado, debido a que la zona del estudio resultó ser hiperendémica, los resultados no serían extrapolables a otras realidades.

Podemos concluir que el porcentaje de infección con equinococosis y la fertilidad quística en porcinos faenados en Huancarama son de los más altos en Perú y el mundo. Los porcinos adultos tuvieron más oportunidad de presentar quistes, al igual que los provenientes de Andahuaylas. Los quistes pulmonares fueron menos frecuentes. El porcentaje de fertilidad entre quistes hepáticos y pulmonares fue similar. Las hembras tuvieron más oportunidad de presentar quistes hepáticos fértiles, al igual que los porcinos adultos. Los quistes de mayor tamaño presentaron mayor porcentaje de fertilidad.

Contribuciones de autoría: RSR ha participado en la recolección, análisis e interpretación de datos. AVP ha participado en la concepción, análisis e interpretación de datos, redacción, diseño, revisión crítica del artículo, asesoría estadística y asesoría técnica o administrativa. Ambos aprobaron la versión final.

Fuente de financiamiento: la investigación fue autofinanciada por los autores.

Conflictos de interés: los autores declaran no tener conflictos de interés en la publicación de este artículo.

\section{REFERENCIAS BIBLIOGRÁFICAS}

1. Acha P, Szyfres B. Zoonosis y enfermedades transmisibles comunes al hombre y los animales. 3a ed. Washington DC: Organización Panamericana de la Salud; 2003.

2. Irabedra P, Salvatella R. El proyecto subregional cono sur de control y vigilancia de la hidatidosis. Rev Peru Med Exp Salud Pública. 2010;27(4):598-603.

3. Organización Mundial de Sanidad Animal. Fichas de información general sobre enfermedades animales: Equinococosis o hidatidosis [internet]. Paris, Francia: OIE; 2011 [citado el 3 de septiembre de 2016]. Disponible en: http: www.oie.int/doc/ged/D13942.PDF

4. Valderrama AA, Huaranca E. Conocimientos y prácticas como factores de riesgo de hidatidosis en animales de Huancarama, Perú. Revista del Colegio de Médicos Veterinarios del Estado Lara. 2014;1(7).

5. Náquira C. Las zoonosis parasitarias en el Perú, su impacto en la economía y salud del país. Academia Nacional de Medicina-Anales. 2006;124-27.

6. Muñoz JP, Sievers G. Estudio de la fertilidad y viabilidad de quistes hidatídicos bovinos en Chile. Parasitol Latinoam. 2005;60:69-73.

7. Instituto Nacional de Estadística e Informática [Internet]. Lima:INEI; 2016 [Citado el 2 de setiembre de 2016] Disponible en: http://www.inei. gob.pe

8. Valderrama AA. Evaluación de la prevención de hidatidosis en el matadero municipal de Abancay, Perú. Revista del Colegio de Médicos Veterinarios del Estado Lara. 2015; $5(2): 10$
9. Ministerio de Agricultura. Reglamento Sanitario del Faenado de Animales de Abasto [internet]. Lima: SENASA; 2012 [citado el 5 de setiembre de 2016]. Disponible en: http:// minagri.gob.pe/portal/download/ $\mathrm{pdf} / \mathrm{marcolegal} /$ normaslegales/ decretossupremos/2012/reglam ds015-2012.pdf.

10. Martínez Revilla M, Galarza Porras E, Rodríguez Vela J, Leguía Puente G, Montes Rojas G. Prevalencia y fertilidad de quistes hidatídicos en ovinos de raza Junín y echinococcosis canina en una ganadería de la sierra central del país. Rev perú Parásitol. 2002;16(1):14-17.

11. Moro PL, McDonald J, Gilman RH, Silva B, Verastegui M, Malqui V, et al. Epidemiology of Echinococcus granulosus infection in the central Peruvian Andes. Bull World Health Organ. 1997;75(6):553-61.

12. Vargas Rivera I, Martínez Maya, JJ, Jaramillo Arango CJ. Caracterización de la hidatidosis porcina en el rastro Los Reyes La Paz, Estado de México. Vet Méx. 1995;26(4):365-8.

13. Negro PS, Ardusso GL, Pagano FG, Bonifacio DR, Bassi AR, Giudici CJ, et al. Caracterización del quiste hidatídico en la especie porcina. Rev Med Vet (Buenos Aires). 2007;88(6):237-241.

14. Rosas Hinostroza HP. Prevalencia de lesiones macroscópicas de hidatidosis durante el periodo 20022006 en mataderos de la provincia de Osorno, Chile. Rev electrón vet. 2010;11(12):1-15.

15. Sánchez Romaní E, Cáceres Rey O, Náquira Velarde C. Polimorfismo genético de Echinococcus granulosus aislados de diferentes hospederos de áreas endémicas del Perú [internet]. Lima: Instituto Nacional de Salud; 2002. Informe técnico $\mathrm{N}^{\circ} 62.2002$.

16. Sarma MD, Deka DK, Borkakoty MR. Occurrence of hydatidosis and porcine cysticercosis in Guwahati city. Journal of Veterinary Parasitology. 2000;14(2):173-4.

17. Ndirangu PN, Njeruh FM, Gathura PB, Kyule MN. The Potential Role Played by Various Livestock Intermediate Hosts in the Transmission of Hydatidosis in Kenya. The Kenya Veterinarian. 2004;27:77-82.

18. Turčeková L', Šnábel V, Dudiňák V, Gašpar V, Dubinský P. Prevalence of cystic echinococcosis in pigs from Slovakia, with evaluation of size, fertility and number of hydatid cysts. Helminthology. 2009; 46(3):151-8.

19. Larrieu E, Belloto A, Arambulo P, Tamayo H. Echinococcosis quística: epidemiología y control en América del Sur. Parasitol Latinoam. 2004;59(12):82-9.

20. Paredes R, Godoy P, Rodríguez B, García MP, Cabezón C, Cabrera G, et al. Bovine (Bos taurus) Humoral Immune Response Against Echinococcus granulosus and Hydatid Cyst Infertility. J Cell Biochem. 2011;112(1):189-99. doi: 10.1002/jcb.22916.

Correspondencia: Aldo Alim Valderrama Pomé

Dirección: Pasaje 9 de agosto s/n; Abancay. Apurimac, Perú.

Correo electrónico:alimvalderrama@gmail.com 